\title{
Nonconforming FEMs for the $p$-Laplace Problem
}

\author{
D. J. Liu*, A. Q. Li and Z. R. Chen \\ Department of Mathematics, Shanghai University, Shanghai 200444, China
}

Received 3 May 2018; Accepted (in revised version) 8 July 2018

\begin{abstract}
The $p$-Laplace problems in topology optimization eventually lead to a degenerate convex minimization problem $E(v):=\int_{\Omega} W(\nabla v) d x-\int_{\Omega} f v d x$ for $v \in W_{0}^{1, p}(\Omega)$ with unique minimizer $u$ and stress $\sigma:=D W(\nabla u)$. This paper proposes the discrete Raviart-Thomas mixed finite element method (dRT-MFEM) and establishes its equivalence with the Crouzeix-Raviart nonconforming finite element method (CR-NCFEM). The sharper quasi-norm a priori and a posteriori error estimates of this two methods are presented. Numerical experiments are provided to verify the analysis.
\end{abstract}

AMS subject classifications: 65N12, 65N30, 65Y20

Key words: Adaptive finite element methods, nonconforming, $p$-Laplace problem, dual energy.

\section{Introduction}

We consider the following nonlinear $p$-Laplace problem $(2 \leq p<\infty)$ in the bounded Lipschitz domain $\Omega \subset \mathbb{R}^{2}$ with the given $f \in L^{q}(\Omega)$ ( $q$ conjugate of $p$ ),

$$
\begin{cases}-\operatorname{div}\left(|\nabla u|^{p-2} \nabla u\right)=f & \text { in } \Omega, \\ u=0 & \text { on } \partial \Omega .\end{cases}
$$

This type of equation appears in many mathematical models of physical process, nonlinear diffusion and filtration, power-law materials, and viscoelastic materials, see $[18,27]$ for example. Most of these mathematical modeling are equivalent to the convex minimization problem [15] with energy

$$
E(v):=\int_{\Omega} W(\nabla v) d x-F(v) \quad \text { for } v \in V:=W_{0}^{1, p}(\Omega)=\left\{v \in W^{1, p}(\Omega):\left.v\right|_{\partial \Omega}=0\right\} .
$$

${ }^{*}$ Corresponding author.

Email: liudj@shu.edu.cn (D. J. Liu) 
Here and throughout this paper, $F(v):=\int_{\Omega} f v d x$ and the energy density function $W: \mathbb{R}^{2} \rightarrow$ $\mathbb{R}$ reads $W(A):=|A|^{p} / p$ with the derivative $D W(A)=|A|^{p-2} A$ for all $A \in \mathbb{R}^{2} \backslash\{0\}$ and the dual function

$$
W^{*}(A):=\frac{|A|^{q}}{q} \quad\left(\frac{1}{p}+\frac{1}{q}=1\right)
$$

Finite element approximation for (1.1) has been extensively studied by many authors, the previous works on a priori and a posteriori error estimations in the conventional $W^{1, p}(\Omega)$-norm can be found, for example, in $[15,16,18,25,28]$. Sharper a priori error estimates were derived in $[4,17,20]$ by developing the quasi-norm techniques, and these techniques were extended to establish improved a posteriori error estimators of residual type for the $\mathcal{P}_{1}$ conforming finite element methods (CFEM) and nonconforming finite element methods (NCFEM) [12,14,21,22]. In [19], Kim applied quasinorm techniques to a mixed finite volume method. Nevertheless, the NCFEM analysis of flux $\sigma:=D W(\nabla u)$, which is important in physical process and also the topic here, is almost not covered in the above references.

This paper focuses on (1.2) and the analysis of flux $\sigma$, proposes some simplified mixed finite element method (MFEM) with one-point numerical quadrature and explores some surprising advantages of the novel discrete Raviart-Thomas mixed finite element method (dRT-MFEM). First, the dRT-MFEM is equivalent to the Crouzeix-Raviart nonconforming first-order finite element method (CR-NCFEM). This generalizes the Marini representation $[3,24]$ and Arbogast [2] from linear and general variable coefficients elliptic PDEs to nonlinear $p$-Laplace problems. Second, the quasi-norm convergence analysis of dRTMFEM (CR-NCFEM) leads to some optimal convergence rates with effective a posteriori error control.

The remaining parts of this paper are organized as follows. Section 2 introduces the precise notation and states the CR-NCFEM and dRT-MFEM for the $p$-Laplace problem. Section 3 establishes the equivalence result of dRT-MFEM and CR-NCFEM. The quasinorm a priori and a posteriori error estimates of CR-NCFEM and dRT-MFEM follow in Section 4 and Section 5. Some numerical experiments conclude the paper in Section 6 with empirical evidence of the superiority of the new NCFEM also for adaptive meshrefinement.

Standard notation applies throughout this paper to Lebesgue and Sobolev spaces $L^{p}(\Omega), H^{s}(\Omega)$, and $H(\operatorname{div}, \Omega)$, as well as to the associated norms $\|\cdot\|_{p, \Omega}:=\|\cdot\|_{L^{p}(\Omega)}$, $\|\mid \cdot\|_{p, \Omega}:=\|\nabla \cdot\|_{L^{p}(\Omega)}$, and $\|\cdot \mid\|_{N C, p, \Omega}:=\left\|\nabla_{N C} \cdot\right\|_{L^{p}(\Omega)}$ with the piecewise gradient $\left.\nabla_{N C} \cdot\right|_{T}:=$ $\nabla\left(\left.\cdot\right|_{T}\right)$ for all $T$ in a regular triangulation $\mathcal{T}$ of the polygonal Lipschitz domain $\Omega$. Here and throughout, ":" denotes the scalar product in $\mathbb{R}^{m \times n}$ and the expression " $\lesssim$ " abbreviates an inequality up to some multiplicative generic constant, i.e., $A \lesssim B$ means $A \leq C B$ with some generic constant $0 \leq C<\infty$, which depends on the interior angles of the triangles but not their sizes. 


\section{Nonconforming FEMs for $p$-Laplace problem}

\subsection{Triangulations}

Let $\mathcal{T}$ be a regular triangulation of the simply-connected bounded Lipschitz domain $\Omega \subseteq \mathbb{R}^{2}$ with polygonal boundary $\partial \Omega$ into closed triangles. That is, the intersection of two distinct and non-disjoint triangles is either a common node or a common edge. Let $\mathcal{E}$ denote the set of all edges and $\mathcal{E}(\Omega)($ resp. $\mathcal{E}(\partial \Omega))$ denote the set of all interior (resp. boundary) edges, $\mathcal{N}$ denote the set of vertices and $\mathcal{N}(\Omega)($ resp. $\mathcal{N}(\partial \Omega)$ ) denote the interior (resp. boundary) nodes. For any triangle $T \in \mathcal{T}$, set $h_{T}:=\operatorname{diam}(T)$ and let $\mathcal{E}(T)$ denote the set of three edges of $T$, write $h_{E}:=\operatorname{diam}(E)$ for an edge $E \in \mathcal{E}(T)$. Let

$$
\mathcal{P}_{k}(\mathcal{T})=\left\{v_{k}: \Omega \rightarrow \mathbb{R}\left|\forall T \in \mathcal{T}, v_{k}\right|_{T} \text { is a polynomial of total degree } \leq k\right\}
$$

denote the set of piecewise polynomials and let $h_{\mathcal{T}} \in \mathcal{P}_{0}(\mathcal{T})$ denote the $\mathcal{T}$-piecewise constant mesh size function with $\left.h_{\mathcal{T}}\right|_{T}=h_{T}$ for all $T \in \mathcal{T}$ and the maximum $h_{\max }:=\left\|h_{\mathcal{T}}\right\|_{\infty}$. Assume that $\mathcal{T}$ is shape-regular so that $h_{T} \approx h_{E} \approx|T|^{1 / 2}$ for all $E \in \mathcal{E}(T)$ and $T \in \mathcal{T}$.

Let $[\cdot]_{E}:=\left.\cdot\right|_{T_{+}}-\left.\cdot\right|_{T_{-}}$denote the jump across the common edge $E=\partial T_{+} \cap \partial T_{-}$with $T_{+}, T_{-} \in \mathcal{T}$ and unit normal $v_{E}$ pointing into $T_{-}$. Let $\Pi_{0}: L^{q}(\Omega) \rightarrow \mathcal{P}_{0}(\mathcal{T})$ denote the $L^{q}$ projection onto $\mathcal{T}$ piecewise constant, i.e., $\left.\left(\Pi_{0} f\right)\right|_{T}=f_{T} f d x$ for all $T \in \mathcal{T}$ (the same notation $\Pi_{0}$ is also used for vectors and understood componentwise), and let $\operatorname{osc}(f, \mathcal{T}):=\| h_{\mathcal{T}}(f-$ $\left.\Pi_{0} f\right) \|_{q, \Omega}$.

\subsection{Crouzeix-Raviart nonconforming FEM}

The Crouzeix-Raviart finite element space is defined as

$$
\begin{aligned}
C R_{0}^{1}(\mathcal{T}):=\left\{v_{h}\right. & \in \mathcal{P}_{1}(\mathcal{T}) \mid v_{h} \text { is continuous at midpoints of interior } \\
& \text { edges and vanishes at midpoints of boundary edges }\} .
\end{aligned}
$$

The nonconforming FEM is based on $C R_{0}^{1}(\mathcal{T})$ and the nonconforming energy $E_{\mathrm{NC}}$ with $F_{h}(\cdot):=F \circ \Pi_{0}(\cdot)=\int_{\Omega}\left(\Pi_{0} f\right) \cdot d x$ and

$$
E_{N C}\left(v_{C R}\right):=\int_{\Omega} W\left(\nabla_{N C} v_{C R}\right) d x-F_{h}\left(v_{C R}\right) \quad \text { for } v_{C R} \in C R_{0}^{1}(\mathcal{T}) .
$$

The Crouzeix-Raviart finite element approximation $u_{C R}$ to (1.2) minimizes the energy $E_{N C}$ in $C R_{0}^{1}(\mathcal{T})$, written

$$
u_{C R} \in \operatorname{argmin} E_{N C}\left(C R_{0}^{1}(\mathcal{T})\right)
$$

The discrete stress $\sigma_{C R}:=D W\left(\nabla_{N C} u_{C R}\right)$ is unique, which will be proven in Section 3, while an a priori and a posteriori error analysis follows in Section 4. 


\subsection{Discrete Raviart-Thomas mixed FEM}

The dual energy $E^{*}$ is defined as

$$
E^{*}(\tau):=-\int_{\Omega} W^{*}(\tau) d x \quad \text { for } \tau \in L^{q}\left(\Omega ; \mathbb{R}^{2}\right) .
$$

Here and throughout this paper, $W^{*}(A):=\sup _{B \in \mathbb{R}^{2}}(A \cdot B-W(B))$ denotes the dual of $W$ [26] and reads as (1.3). The dual problem of (1.2) maximizes the energy $E^{*}$ in $Q(f):=$ $\{\tau \in H(\operatorname{div}, \Omega) \mid f+\operatorname{div}(\tau)=0$ a.e. in $\Omega\}$, written

$$
\sigma=\operatorname{argmax} E^{*}(Q(f)) .
$$

The maximizer $\sigma$ is unique [18] and $\sigma:=D W(\nabla u)$ for minimizer $u$ of $E$ in $W_{0}^{1, p}(\Omega)$.

Define the Raviart-Thomas finite element space

$$
R T_{0}(\mathcal{T}):=\left\{p \in H(\operatorname{div}, \Omega) \mid \forall T \in \mathcal{T}, \exists a \in \mathbb{R}^{2}, b \in \mathbb{R}, \forall x \in T, p=a+b x\right\}
$$

and $Q(f, \mathcal{T}):=\left\{\tau_{R T} \in R T_{0}(\mathcal{T}) \mid \Pi_{0} f+\operatorname{div}\left(\tau_{R T}\right)=0\right.$ a.e. in $\left.\Omega\right\}$. The discrete Raviart-Thomas mixed finite element scheme is based on the one-point numerical quadrature with respect to the center of each triangle and the resulting discrete dual energy $E_{d}^{*}:=E^{*} \circ \Pi_{0}$,

$$
E_{d}^{*}\left(\tau_{R T}\right)=-\int_{\Omega} W^{*}\left(\Pi_{0} \tau_{R T}\right) d x \text { for } \tau_{R T} \in Q(f, \mathcal{T}) .
$$

The discrete Raviart-Thomas mixed finite element approximation $\sigma_{d R T}$ to the dual solution $\sigma$ maximizes the energy $E_{d}^{*}$ in $Q(f, \mathcal{T})$, written

$$
\sigma_{d R T}=\operatorname{argmax} E_{d}^{*}(Q(f, \mathcal{T})) .
$$

The strong convexity of $W^{*}$ (see Lemma 3.3 below) shows that the maximizer $\sigma_{d R T}$ is unique in $Q(f, \mathcal{T})$. An a priori and a posteriori error analysis follows in Section 5.

\section{CR-NCFEM is equal to dRT-MFEM}

This section is devoted to the equivalence of CR-NCFEM from Subsection 2.2 with dRTMFEM from Subsection 2.3 as a generalization of the Marini representation from the linear equations $[2,3,24]$ to nonlinear convex minimization problems. The equivalence is expressed by the equivalence of $\sigma_{d R T}$ with some post-processing $\sigma_{C R}^{*}$ of $\sigma_{C R}$, namely

$$
\sigma_{C R}^{*}:=\sigma_{C R}-\frac{\Pi_{0} f}{2}(\cdot-\operatorname{mid}(\mathcal{T})) \in \mathcal{P}_{1}\left(\mathcal{T} ; \mathbb{R}^{2}\right)
$$

Here and throughout this paper, the piecewise affine function $\cdot-\operatorname{mid}(\mathcal{T}) \in \mathcal{P}_{1}(\mathcal{T})$ equals $x-\operatorname{mid}(T)$ at $x \in T \in \mathcal{T}$ with barycenter $\operatorname{mid}(T)$. 
Theorem 3.1 (CR-NCFEM $=$ dRT-MFEM with no discrete duality gap). It holds $\sigma_{C R}^{*}=\sigma_{d R T}$ and $\max E_{d}^{*}(Q(f, \mathcal{T}))=\min E_{N C}\left(C R_{0}^{1}(\mathcal{T})\right)$.

The remaining parts of this section are devoted to the proof of Theorem 3.1 which is based on the following lemmas and the Crouzeix-Raviart interpolation operator $I_{N C}$ : $W_{0}^{1, p}(\Omega) \rightarrow C R_{0}^{1}(\mathcal{T}),(2 \leq p \leq \infty)$,

$$
\left(I_{N C} v\right)(\operatorname{mid}(E)):=f_{E} v d s \text { for all } E \in \mathcal{E} .
$$

Lemma 3.1 (Property of the Crouzeix-Raviart interpolant, see $[9,10,15]) . A n y v \in W^{1, p}(\Omega)$ with its interpolation $I_{N C} v$ and the constant $\kappa$ satisfy $\nabla_{N C}\left(I_{N C} v\right)=\Pi_{0} \nabla v$ and

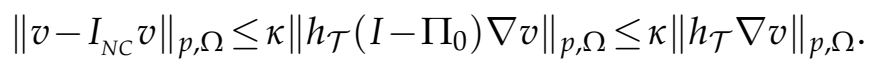

Lemma 3.2 (Conforming $\mathcal{P}_{3}$ companion, see [13]). Given any $v_{C R} \in C R_{0}^{1}(\mathcal{T})$, there exists some $v_{3} \in \mathcal{P}_{3}(\mathcal{T}) \cap W_{0}^{1, p}(\Omega)$ with $v_{C R}=I_{N C} v_{3}, \Pi_{0} v_{C R}=\Pi_{0} v_{3}$, and

$$
\left\|h_{\mathcal{T}}^{-1}\left(v_{C R}-v_{3}\right)\right\|_{p, \Omega}+\left|\left\|v_{C R}-v_{3}\left|\left\|\left._{N C, p, \Omega} \lesssim \min _{v \in V}\left|\| v-v_{C R}\right|\right|_{N C, p, \Omega} .\right.\right.\right.\right.
$$

The subdifferential $\partial W^{*}$ of $W^{*}[26]$ is uniformly convex.

Lemma 3.3. Given $2 \leq p<\infty$ and the conjugate $q$, there exists a positive constant $c(p)$ such that for any $a, b \in \mathbb{R}^{2} \backslash\{0\}, \alpha:=D W(a), \beta:=D W(b)$ satisfy

$$
\frac{1}{\left(|\alpha|^{2-q}+|\beta|^{2-q}\right)}|\alpha-\beta|^{2} \leq c(p)(W(b)-W(a)-\alpha \cdot(b-a)) .
$$

Any $\alpha, \beta \in \mathbb{R}^{2} \backslash\{0\}$ and any $b \in \partial W^{*}(\beta)$ satisfy

$$
\frac{1}{\left(|\alpha|^{2-q}+|\beta|^{2-q}\right)}|\alpha-\beta|^{2} \leq c(p)\left(W^{*}(\alpha)-W^{*}(\beta)-b \cdot(\alpha-\beta)\right) .
$$

Proof. Given $a, b \in \mathbb{R}^{2} \backslash\{0\}$ with $a \neq b$, set $t:=|b| /|a|$ and $g:=a: b /(|a| \cdot|b|)(-1 \leq g \leq 1)$, the paper [6, Lemma3.1] shows

$$
\begin{aligned}
& \frac{1}{\left(|a|^{p-2}+|b|^{p-2}\right)(W(b)-W(a)-\alpha \cdot(b-a))}|\alpha-\beta|^{2} \\
= & \frac{1+t^{2(p-1)}-2 g t^{p-1}}{\left(1+t^{p-2}\right)\left(t^{p} / p+1 / q-g t\right)}:=f(t, g) .
\end{aligned}
$$

The formula $D W(a)=|a|^{p-2} a$ implies that $|\alpha|=|a|^{p-1}$ and $|\beta|=|b|^{p-1}$. The combination with conjugate property leads to $|a|^{p-2}=|\alpha|^{2-q}$ and $|b|^{p-2}=|\beta|^{2-q}(0 \leq 2-q<1)$, and the left side of (3.3) is rewritten as

$$
\frac{1}{\left(|\alpha|^{2-q}+|\beta|^{2-q}\right)(W(b)-W(a)-\alpha \cdot(b-a))}|\alpha-\beta|^{2} .
$$


A direct calculation verifies that $\partial f / \partial g$ as a function of $g$ has one sign (which depends on $t$ and $p$ ), hence it is monotone increasing or decreasing. Therefore for all $0<t<\infty$, there exists a constant $c(p)$ satisfies

$$
\min \{f(t, 1), f(t,-1)\} \leq c(p):=\max \{f(t, 1), f(t,-1)\}<\infty .
$$

The case $g=1$ is the crucial one because $t^{p} / p+1 / q-t$ vanishes for $t=1$,

$$
f(t, 1)=\frac{\left(1-t^{p-1}\right)^{2}}{\left(1+t^{p-2}\right)\left(t^{p} / p+1 / q-t\right)} .
$$

L'Hospital rule yields $f(1,1)=p-1>0$. The monotone decreasing and monotone increasing of $t^{p} / p+1 / q-t$ on $(0,1)$ and $(1, \infty)$ show that $t^{p} / p+1 / q-t>0$, that is $f(t, 1)>0$. The analysis of $f(t,-1)>0$ is simpler and hence omitted, hence $c(p)>0$. The (3.1) is proved, which is also known as convexity control of $W$.

The duality in convex analysis shows that the relation $\alpha=D W(a)$ is equivalent to $W^{*}(\alpha)+W(a)=a \cdot \alpha[26$, Theorem 23.5]. This implies

$$
W^{*}(\alpha)+W(a)=a \cdot \alpha \quad \text { and } \quad W^{*}(\beta)+W(b)=b \cdot \beta .
$$

The combination with (3.1) concludes the proof of (3.2).

Remark 3.1. The basic calculation can prove that $0<c(p)<2 p$.

Define the weighed norm

$$
|\langle\alpha, \beta\rangle|_{q}:=\sqrt{\int_{\Omega} \frac{1}{\left(|\alpha|^{2-q}+|\beta|^{2-q}\right)}|\alpha-\beta|^{2} d x} .
$$

The following lemma shows that the defined norm (3.4) is a quasi-norm

Lemma 3.4. It holds that

(i) $|\langle\alpha, \beta\rangle|_{q} \geq 0$, and $|\langle\alpha, \beta\rangle|_{q}=0$ if and only if $\alpha=\beta$.

(ii) $\forall a_{1}, a_{2}, b_{1}, b_{2} \in \mathbb{R}^{2} \backslash\{0\}, \alpha_{1}:=D W\left(a_{1}\right), \beta_{1}:=D W\left(b_{1}\right), \alpha_{2}:=D W\left(a_{2}\right), \beta_{2}:=D W\left(b_{2}\right)$, $\left|\left\langle\alpha_{1}+\alpha_{2}, \beta_{1}+\beta_{2}\right\rangle\right|_{q} \leq 2^{\frac{q-1}{2}}\left(\left|\left\langle\alpha_{1}, \beta_{1}\right\rangle\right|_{q}+\left|\left\langle\alpha_{2}, \beta_{2}\right\rangle\right|_{q}\right)$.

Proof. (i) According to the expression, it is easy to prove that $|\langle\alpha, \beta\rangle|_{q} \geq 0 .|\langle\alpha, \beta\rangle|_{q}=0$, that is

$$
\left(\int_{\Omega} \frac{|\alpha-\beta|^{2}}{|\alpha|^{2-q}+|\beta|^{2-q}} d x\right)^{\frac{1}{2}}=0
$$

if and only if $\alpha=\beta$.

(ii) For $\forall x, y \in \mathbb{R}^{2} \backslash\{0\}$, define

$$
f(x, y)=\frac{|x-y|^{2}}{|x|^{m}+|y|^{m}}, \quad(0 \leq m<1) .
$$


A direct calculation shows that

$$
\left\{\begin{array}{c}
f_{x x}=\frac{2 m^{2} x^{2}|x|^{2 m-4}(x-y)^{2}}{\left(|x|^{m}+|y|^{m}\right)^{3}}+\frac{2}{|x|^{m}+|y|^{m}}-\frac{2 m x|x|^{m-2}(2 x-2 y)}{\left(|x|^{m}+|y|^{m}\right)^{2}} \\
-\frac{m(m-1) x^{2}|x|^{m-4}(x-y)^{2}}{\left(|x|^{m}+|y|^{m}\right)^{2}}, \\
f_{x y}=\frac{m x|x|^{m-2}(2 x-2 y)}{\left(|x|^{m}+|y|^{m}\right)^{2}}-\frac{2}{|x|^{m}+|y|^{m}}-\frac{m y|y|^{m-2}(2 x-2 y)}{\left(|x|^{m}+|y|^{m}\right)^{2}} \\
+\frac{2 m^{2} x|x|^{m-2} y|y|^{m-2}(x-y)^{2}}{\left(|x|^{m}+|y|^{m}\right)^{3}}, \\
f_{y y}=\frac{2 m^{2} y^{2}|y|^{2 m-4}(x-y)^{2}}{\left(|x|^{m}+|y|^{m}\right)^{3}}+\frac{2}{|x|^{m}+|y|^{m}}+\frac{2 m y|y|^{m-2}(2 x-2 y)}{\left(|x|^{m}+|y|^{m}\right)^{2}} \\
-\frac{m(m-1) y^{2}|y|^{m-4}(x-y)^{2}}{\left(|x|^{m}+|y|^{m}\right)^{2}} .
\end{array}\right.
$$

We can rewrite $f_{x x}$ and $f_{x x} \cdot f_{y y}-f_{x y}^{2}$ as

$$
\begin{array}{r}
f_{x x}=\frac{1}{\left(\left(|x|^{m}+|y|^{m}\right)\right)^{3}}\left\{2\left[m x|x|^{m-2}(x-y)-\left(|x|^{m}+|y|^{m}\right)\right]^{2}\right. \\
\left.-m(m-1) x^{2}|x|^{m-4}(x-y)^{2}\left(|x|^{m}+|y|^{m}\right)\right\}
\end{array}
$$

and

$$
\begin{aligned}
& f_{x x} \cdot f_{y y}-f_{x y}^{2} \\
= & \frac{1}{\left(|x|^{m}+|y|^{m}\right)^{6}}\left\{m^{2}(m-1)^{2} x^{2}|x|^{m-4} y^{2}|y|^{m-4}(x-y)^{4}\right. \\
& \quad-2 m(m-1) x^{2}|x|^{m-4}\left(|x|^{m}+|y|^{m}\right)(x-y)^{2}\left[m y|y|^{m-2}(x-y)+\left(|x|^{m}+|y|^{m}\right)\right]^{2} \\
& \left.\quad-2 m(m-1) y^{2}|y|^{m-4}\left(|x|^{m}+|y|^{m}\right)(x-y)^{2}\left[m x|x|^{m-2}(x-y)-\left(|x|^{m}+|y|^{m}\right)\right]^{2}\right\} .
\end{aligned}
$$

Since $0 \leq m<1$ and $-m(m-1)>0$, hence $f_{x x}>0, f_{x x} \cdot f_{y y}-f_{x y}^{2}>0$, which imply that the $f(x, y)$ is a convex function.

Take $x:=\alpha, y:=\beta$ in (3.5) and the Jensen's inequality shows that

$$
\begin{aligned}
& \int_{\Omega} \frac{1}{\left|\frac{\alpha_{1}+\alpha_{2}}{2}\right|^{m}+\left|\frac{\beta_{1}+\beta_{2}}{2}\right|^{m}}\left|\frac{\alpha_{1}+\alpha_{2}}{2}-\frac{\beta_{1}+\beta_{2}}{2}\right|^{2} d x \\
\leq & \frac{1}{2}\left[\int_{\Omega} \frac{\left|\alpha_{1}-\beta_{1}\right|^{2}}{\left|\alpha_{1}\right|^{m}+\left|\beta_{1}\right|^{m}} d x+\int_{\Omega} \frac{\left|\alpha_{2}-\beta_{2}\right|^{2}}{\left|\alpha_{2}\right|^{m}+\left|\beta_{2}\right|^{m}} d x\right] .
\end{aligned}
$$


That is

$$
\begin{aligned}
& \int_{\Omega} \frac{1}{\left|\alpha_{1}+\alpha_{2}\right|^{m}+\left|\beta_{1}+\beta_{2}\right|^{m}}\left|\left(\alpha_{1}+\alpha_{2}\right)-\left(\beta_{1}+\beta_{2}\right)\right|^{2} d x \\
\leq & \frac{1}{2^{m-1}}\left[\int_{\Omega} \frac{\left|\alpha_{1}-\beta_{1}\right|^{2}}{\left|\alpha_{1}\right|^{m}+\left|\beta_{1}\right|^{m}} d x+\int_{\Omega} \frac{\left|\alpha_{2}-\beta_{2}\right|^{2}}{\left|\alpha_{2}\right|^{m}+\left|\beta_{2}\right|^{m}} d x\right] .
\end{aligned}
$$

The combination of (3.4) and $m:=2-q$ in (3.6) concludes the proof.

Lemma 3.5 (Uniqueness of $\sigma_{C R}$ ). The discrete stress $\sigma_{C R}$ is unique and satisfies the discrete Euler-Lagrange equation in the sense that

$$
\int_{\Omega} \sigma_{C R} \cdot \nabla_{N C} v_{C R} d x=\int_{\Omega}\left(\Pi_{0} f\right) v_{C R} d x \quad \text { for } v_{C R} \in C R_{0}^{1}(\mathcal{T}) .
$$

Proof. For any $0<\varepsilon<1$ and any $v_{C R} \in C R_{0}^{1}(\mathcal{T})$, let

$$
\delta_{\varepsilon}(x):=\frac{W\left(\nabla_{N C} u_{C R}(x)+\varepsilon \nabla_{N C} v_{C R}(x)\right)-W\left(\nabla_{N C} u_{C R}(x)\right)}{\varepsilon} \text { for all } x \in \Omega .
$$

Since $u_{C R}$ is a minimizer,

$$
0 \leq \frac{E_{N C}\left(u_{C R}+\varepsilon v_{C R}\right)-E_{N C}\left(u_{C R}\right)}{\varepsilon}=\int_{\Omega} \delta_{\varepsilon}(x) d x-F_{h}\left(v_{C R}\right) .
$$

Since $W$ is smooth, it follows for almost every $x \in \Omega$, that

$$
\begin{aligned}
\left|\delta_{\varepsilon}(x)\right| & =\left|\frac{1}{\varepsilon} \int_{0}^{1} \frac{D W\left(\nabla_{N C} u_{C R}(x)+\varepsilon s \nabla_{N C} v_{C R}(x)\right)}{\partial s} d s\right| \\
& \leq \int_{0}^{1}\left|D W\left(\nabla_{N C} u_{C R}(x)+\varepsilon s \nabla_{N C} v_{C R}(x)\right) \cdot \nabla_{N C} v_{C R}(x)\right| d s .
\end{aligned}
$$

The formula $|D W(A)|=\left.\left.|| A\right|^{p-2} A|=| A\right|^{p-1}$ and the Young inequality imply that

$$
\begin{aligned}
\left|\delta_{\varepsilon}(x)\right| & \leq \int_{0}^{1}\left|\nabla_{N C} u_{C R}(x)+\varepsilon s \nabla_{N C} v_{C R}(x)\right|^{p-1}\left|\nabla_{N C} v_{C R}(x)\right| d s \\
& \lesssim\left|\nabla_{N C} v_{C R}(x)\right|^{p}+\left|\nabla_{N C} u_{C R}(x)\right|^{p} .
\end{aligned}
$$

The Lemma 3.2 imply that $\int_{\Omega}\left(\left|\nabla_{N C} v_{C R}(x)\right|^{p}+\left|\nabla_{N C} u_{C R}(x)\right|^{p}\right) d x$ exists, hence the Lebesgue dominate convergence theorem guarantees

$$
\lim _{\varepsilon \rightarrow 0} \int_{\Omega} \delta_{\varepsilon}(x) d x=\int_{\Omega} D W\left(\nabla_{N C} u_{C R}\right) \cdot \nabla_{N C} v_{C R} d x
$$

This and (3.7) imply

$$
0 \leq \int_{\Omega} D W\left(\nabla_{N C} u_{C R}\right) \cdot \nabla_{N C} v_{C R} d x-F_{h}\left(v_{C R}\right)
$$


Since $v_{C R}$ is arbitrary in $C R_{0}^{1}(\mathcal{T})$, this proves the asserted discrete Euler-Lagrange equation.

The uniqueness of $u_{C R}$ leads to the uniqueness of the stress $\sigma_{C R}=D W\left(\nabla_{N C} u_{C R}\right)$. This concludes the proof.

Lemma 3.6 (see [13]). It holds $\sigma_{C R}^{*} \in Q(f, \mathcal{T}) \subseteq H(\operatorname{div}, \Omega)$.

Proof of Theorem 3.1. For minimizer $u_{C R}$ of (2.2), the duality relation $\sigma_{C R}=D W\left(\nabla_{N C} u_{C R}\right)$ implies that $\nabla_{N C} u_{C R} \in \partial W^{*}\left(\sigma_{C R}\right)$. The choice of $\alpha:=\left.\Pi_{0} \sigma_{d R T}\right|_{T}=\sigma_{d R T}(\operatorname{mid}(T)), \beta:=\Pi_{0} \sigma_{C R}^{*}=$ $\sigma_{C R}$, and $b:=\nabla_{N C} u_{C R}$ in Lemma 3.3 leads to

$$
\begin{aligned}
& \left|\left\langle\Pi_{0} \sigma_{d R T}, \Pi_{0} \sigma_{C R}^{*}\right\rangle\right|_{q}^{2} \\
\leq & c(p) \int_{\Omega} W^{*}\left(\Pi_{0} \sigma_{d R T}\right)-W^{*}\left(\Pi_{0} \sigma_{C R}^{*}\right)-\nabla_{N C} u_{C R} \cdot\left(\sigma_{d R T}-\sigma_{C R}\right) d x \\
\leq & c(p)\left(E^{*}\left(\Pi_{0} \sigma_{C R}^{*}\right)-E^{*}\left(\Pi_{0} \sigma_{d R T}\right)-\int_{\Omega} \nabla_{N C} u_{C R} \cdot\left(\sigma_{d R T}-\sigma_{C R}\right) d x\right) .
\end{aligned}
$$

An integration by parts and Lemma 3.5 with $\sigma_{d R T} \in Q(f, \mathcal{T})$ show that the last term vanishes. This and $E_{d}^{*}:=E^{*} \circ \Pi_{0}$ prove

$$
\left|\left\langle\Pi_{0} \sigma_{d R T}, \Pi_{0} \sigma_{C R}^{*}\right\rangle\right|_{q}^{2} \leq c(p)\left(E_{d}^{*}\left(\sigma_{C R}^{*}\right)-E_{d}^{*}\left(\sigma_{d R T}\right)\right) .
$$

Since $c(p)>0$ for all $p \geq 2, \sigma_{d R T} \in \operatorname{argmax} E_{d}^{*}(Q(f, \mathcal{T}))$ and $\sigma_{C R}^{*} \in Q(f, \mathcal{T})$, the upper bound is non-positive. Hence, $\Pi_{0} \sigma_{d R T}=\sigma_{C R}$ and $E_{d}^{*}\left(\sigma_{C R}^{*}\right)=E_{d}^{*}\left(\sigma_{d R T}\right)$.

The duality relation $\sigma_{C R}=D W\left(\nabla_{N C} u_{C R}\right)$ with the minimizer $u_{C R}$ of (2.2) is equivalent to

$$
W^{*}\left(\sigma_{C R}\right)+W\left(\nabla_{N C} u_{C R}\right)=\sigma_{C R} \cdot \nabla_{N C} u_{C R} \cdot
$$

An integration of this reads

$$
\int_{\Omega} W\left(\nabla_{N C} u_{C R}\right) d x-\int_{\Omega} \sigma_{C R} \cdot \nabla_{N C} u_{C R} d x=-\int_{\Omega} W^{*}\left(\sigma_{C R}\right) d x
$$

The definition of $E_{N C}$ and Lemma 3.5 shows that the left-hand side equals $E_{N C}\left(u_{C R}\right)$. Moreover,

$$
-\int_{\Omega} W^{*}\left(\sigma_{C R}\right) d x=-\int_{\Omega} W^{*}\left(\Pi_{0} \sigma_{d R T}\right) d x=E_{d}^{*}\left(\sigma_{d R T}\right) .
$$

Hence, $E^{*}\left(\sigma_{C R}\right)=E_{d}^{*}\left(\sigma_{d R T}\right)=E_{N C}\left(u_{C R}\right)$. This concludes the proof.

\section{Error analysis of Crouzeix-Raviart NCFEM}

This section analyzes the error estimates of the Crouzeix-Raviart NCFEM. 


\subsection{A priori error analysis}

The following Theorem 4.1 guarantees the convergence estimates of the Crouzeix-Raviart NCFEM.

Theorem 4.1. (A priori error estimate). The discrete stress $\sigma_{C R}$ satisfies

$$
\begin{aligned}
& \quad\left|\left\langle\sigma, \sigma_{C R}\right\rangle\right|_{q}^{2} \\
& \leq c(p) \max \left\{\operatorname{osc}(f, \mathcal{T})\|u\|_{p, \Omega}(\kappa \cdot \operatorname{osc}(f, \mathcal{T})\right. \\
& \left.\left.\quad+\sup _{x \in \Omega}\left(|\sigma|^{1-q / 2}+\left|\Pi_{0} \sigma\right|^{1-q / 2}\right)\left|\left\langle\sigma, \Pi_{0} \sigma\right\rangle\right|_{q}\right)\left\|u-u_{C R} \mid\right\|_{N C, p, \Omega}\right\} .
\end{aligned}
$$

Proof. The choice $a:=\nabla_{N C} u_{C R}, b:=\nabla u$, and $\alpha:=\sigma_{C R}$ in Lemma 3.3 leads to

$$
\left|\left\langle\sigma, \sigma_{C R}\right\rangle\right|_{q}^{2} \leq c(p)\left(E(u)-E_{N C}\left(u_{C R}\right)+F\left(u-u_{C R}\right)-\int_{\Omega} \sigma_{C R} \cdot \nabla_{N C}\left(u-u_{C R}\right) d x\right) .
$$

Since $\sigma_{C R} \in \mathcal{P}_{0}\left(\mathcal{T} ; \mathbb{R}^{2}\right)$ and

$$
\int_{\Omega} \sigma_{C R} \cdot \nabla u d x=\int_{\Omega} \sigma_{C R} \cdot \nabla_{N C} I_{N C} u d x=F_{h}\left(I_{N C} u\right) .
$$

The combination with the previous estimate verifies

$$
\left|\left\langle\sigma, \sigma_{C R}\right\rangle\right|_{q}^{2}+c(p)\left(E_{N C}\left(u_{C R}\right)-E(u)\right) \leq c(p)\left(F(u)-F_{h}\left(I_{N C} u\right)\right) .
$$

The choice $a:=\nabla u, b:=\nabla_{N C} u_{C R}$, and $\alpha:=\sigma$ in Lemma 3.3 leads to

$$
\left|\left\langle\sigma, \sigma_{C R}\right\rangle\right|_{q}^{2}+c(p)\left(E(u)-E_{N C}\left(u_{C R}\right)\right) \leq c(p)\left(F\left(u_{C R}\right)-\int_{\Omega} \sigma \cdot \nabla_{N C} u_{C R} d x\right) .
$$

The conforming $\mathcal{P}_{3}$ companion $u_{3} \in \mathcal{P}_{3}(\mathcal{T}) \cap V$ with $u_{C R}=I_{N C} u_{3}$ shows

$$
\begin{aligned}
-\int_{\Omega} \sigma \cdot \nabla_{N C} u_{C R} d x & =-\int_{\Omega} \sigma \cdot \nabla u_{3} d x+\int_{\Omega} \sigma \cdot \nabla_{N C}\left(u_{3}-I_{N C} u_{3}\right) d x \\
& =-F\left(u_{3}\right)+\int_{\Omega}\left(I-\Pi_{0}\right) \sigma \cdot\left(I-\Pi_{0}\right) \nabla u_{3} d x .
\end{aligned}
$$

The combination of the preceding estimates results in

$$
\begin{aligned}
& \left|\left\langle\sigma, \sigma_{C R}\right\rangle\right|_{q}^{2}+c(p)\left(E(u)-E_{N C}\left(u_{C R}\right)\right) \\
\leq & c(p)\left(F\left(I_{N C} u_{3}-u_{3}\right)+\int_{\Omega}\left(I-\Pi_{0}\right) \sigma \cdot\left(I-\Pi_{0}\right) \nabla u_{3} d x\right) .
\end{aligned}
$$


The (4.1)-(4.2) imply that

$$
\begin{aligned}
& \left|\left\langle\sigma, \sigma_{C R}\right\rangle\right|_{q}^{2}+c(p)\left|E(u)-E_{N C}\left(u_{C R}\right)\right| \\
\leq & c(p) \max \left\{F(u)-F_{h}\left(I_{N C} u\right), F\left(I_{N C} u_{3}-u_{3}\right)+\int_{\Omega}\left(I-\Pi_{0}\right) \sigma \cdot\left(I-\Pi_{0}\right) \nabla u_{3} d x\right\} .
\end{aligned}
$$

The Hölder inequality and Lemma 3.1 prove

$$
\begin{aligned}
& F\left(I_{N C} u_{3}-u_{3}\right)=\int_{\Omega}\left(f-\Pi_{0} f\right)\left(I_{N C} u_{3}-u_{3}\right) d x \leq \kappa \operatorname{osc}(f, \mathcal{T})\left\|u_{3}-I_{N C} u_{3}\right\| \|_{N C, p, \Omega^{\prime}} \\
& \begin{aligned}
F(u)-F_{h}\left(I_{N C} u\right) & =\int_{\Omega} f\left(u-I_{N C} u\right) d x+\int_{\Omega}\left(f-\Pi_{0} f\right) I_{N C} u d x \\
& =\int_{\Omega}\left(f-\Pi_{0} f\right)\left(u-I_{N C} u\right) d x+\int_{\Omega}\left(f-\Pi_{0} f\right) I_{N C} u d x \\
& \leq \operatorname{osc}(f, \mathcal{T})\|u\|_{p, \Omega^{\prime}}
\end{aligned} \\
& \int_{\Omega}\left(I-\Pi_{0}\right) \sigma \cdot\left(I-\Pi_{0}\right) \nabla u_{3} d x \\
& \leq\left|<\sigma, \Pi_{0} \sigma>\right|_{q} \cdot\left(\int_{\Omega}\left(|\sigma|^{2-q}+\left|\Pi_{0} \sigma\right|^{2-q}\right)\left|\left(I-\Pi_{0}\right) \nabla u_{3}\right|^{2} d x\right)^{1 / 2} .
\end{aligned}
$$

This and Lemma 3.2 prove the assertion.

\subsection{A posteriori error analysis}

This subsection is devoted to an a posteriori error analysis of the CR-NCFEM. The error analysis is based on the boundness of minimizers. Recall that any $v \in W_{0}^{1, p}(\Omega)$ satisfies the Friedrichs inequality

$$
\|v\|_{p, \Omega} \leq C_{F}\|v\|_{p, \Omega}
$$

with $C_{F} \leq$ width $(\Omega) / \pi$. Any $v_{C R} \in C R_{0}^{1}(\mathcal{T})$ satisfies the discrete Friedrichs inequality (see [5, pp. 301]) with some constant $C_{d F} \approx 1$

$$
\left\|v_{C R}\right\|_{p, \Omega} \leq C_{d F}\left\|v_{C R}\right\|_{N C, p, \Omega} .
$$

Theorem 4.2 (A posteriori error estimate). The discrete stress $\sigma_{C R}$ and the constants $C_{1}:=$ $2 c(p) C_{\mathcal{P}}^{p-2}\|f\|_{q, \Omega}^{\frac{p-2}{p-1}}$ and $C_{2}:=C_{F} C_{\mathcal{P}}\|f\|_{q, \Omega}^{\frac{1}{p-1}}$ satisfy

$$
\frac{1}{2}\left|\left\langle\sigma, \sigma_{C R}\right\rangle\right|_{q}^{2} \leq c(p) \max \left\{F\left(u_{C R}-u_{3}\right)+C_{1}\left\|u_{C R}-u_{3}\right\|_{N C, 2, \Omega^{\prime}}^{2} C_{2} \cdot \operatorname{osc}(f, \mathcal{T})\right\},
$$

where $C_{\mathcal{P}}:=\left(p \cdot C_{F}\right)^{\frac{1}{p-1}}$. 
Proof. The energy density $W(A)=\frac{|A|^{p}}{p}$ and the Friedrichs inequality shows that

$$
\frac{1}{p}\|u\|_{p, \Omega}^{p}-C_{F}\|f\|_{q, \Omega}\|u\|_{p, \Omega} \leq E(u) .
$$

Since $E(u) \leq E(0)=0$, this implies

$$
\|u\|_{p, \Omega} \leq\left(p C_{F}\|f\|_{q, \Omega}\right)^{\frac{1}{p-1}} .
$$

Recall that $|\nabla u|^{p-2}=|\sigma|^{2-q}$, The estimate (4.3) and the Hölder inequality imply

$$
\begin{gathered}
\left|\left\langle\sigma, \sigma_{C R}\right\rangle\right|_{q}^{2} \\
\leq c(p) \max \left\{C_{F} \cdot \operatorname{osc}(f, \mathcal{T})\|u \mid\|_{p, \Omega}, F\left(u_{C R}-u_{3}\right)\right. \\
\left.\quad+\left.2 \sup _{x \in \Omega}|\nabla u|^{p / 2-1}\left|\left\langle\sigma, \Pi_{0} \sigma\right\rangle\right|_{q}\left\|u_{C R}-u_{3}\right\|\right|_{N C, 2, \Omega}\right\} .
\end{gathered}
$$

The Young inequality shows

$$
\begin{aligned}
& 2 c(p) \sup _{x \in \Omega}|\nabla u|^{p / 2-1}\left|\left\langle\sigma, \Pi_{0} \sigma\right\rangle\right|_{q}|| u_{C R}-u_{3} \mid \|_{N C, 2, \Omega} \\
\leq & \frac{1}{2}\left|\left\langle\sigma, \Pi_{0} \sigma\right\rangle\right|_{q}^{2}+2 c^{2}(p) \sup _{x \in \Omega}|\nabla u|^{p-2}\left\|u_{C R}-u_{3}\right\|_{N C, 2, \Omega}^{2} .
\end{aligned}
$$

The combination of preceding displayed inequalities concludes the proof.

\section{Error analysis of dRT MFEM}

This section analyzes the error of the discrete Raviart-Thomas MFEM.

\subsection{A priori error analysis}

Theorem 3.1 and Theorem 4.1 allow an immediate a priori error estimate.

Theorem 5.1 (A priori error estimate). The discrete stress $\sigma_{d R T}$ satisfies

$$
\begin{aligned}
& \quad\left|\left\langle\sigma, \sigma_{d R T}\right\rangle\right|_{q}^{2} \\
& \leq \frac{1}{2^{1-q}}\left\|h_{\mathcal{T}}\left(\Pi_{0} f\right)\right\|_{q, \Omega}^{q}+\frac{c(p)}{2^{1-q}} \max \left\{\operatorname{osc}(f, \mathcal{T})\|u\|_{p, \Omega}(\kappa \cdot \operatorname{osc}(f, \mathcal{T})\right. \\
& \left.\left.\quad+2 \sup _{x \in \Omega}|\nabla u|^{p / 2-1}\left|\left\langle\sigma, \Pi_{0} \sigma\right\rangle\right|_{q}\right)\left\|u-u_{C R} \mid\right\|_{N C, p, \Omega}\right\}
\end{aligned}
$$


Proof. The Lemma 3.4 and Theorem 3.1 lead to

$$
\begin{aligned}
\left|\left\langle\sigma, \sigma_{d R T}\right\rangle\right|_{q}^{2} & \leq \frac{1}{2^{1-q}}\left(\left|\left\langle\sigma, \sigma_{C R}\right\rangle\right|_{q}^{2}+\left|\left\langle\frac{\Pi_{0} f}{2}(\cdot-\operatorname{mid}(\mathcal{T})), 0\right\rangle\right|_{q}^{2}\right) \\
& \leq \frac{1}{2^{1-q}}\left(\left|\left\langle\sigma, \sigma_{C R}\right\rangle\right|_{q}^{2}+\left\|h_{\mathcal{T}}\left(\Pi_{0} f\right)\right\|_{q, \Omega}^{q}\right) .
\end{aligned}
$$

This and Theorem 4.1 conclude the proof.

The further a posteriori error analysis requires that the arising subgradients are the piecewise gradients of minimizers of $E_{N C}$ in $C R_{0}^{1}(\mathcal{T})$, that is $\Pi_{0}\left(-\partial E_{d}^{*}\left(\sigma_{d R T}\right)\right)=\nabla_{N C} u_{C R}$ (refer to [13]).

\subsection{A posteriori error analysis}

This subsection is devoted to an a posteriori error analysis of the dRT-MFEM.

Theorem 5.2 (A posteriori error estimate). The discrete stress $\sigma_{d R T}$ and the constants $C_{2}:=$ $C_{F} C_{\mathcal{P}}\|f\|_{q, \Omega}^{\frac{1}{p-1}}$ and $M:=C_{\mathcal{P}}^{p-2}\|f\|_{q, \Omega}^{\frac{p-2}{p-1}}+C_{d \mathcal{P}}^{p-2}\|f\|_{q, \Omega}^{\frac{p-2}{p-1}}$ satisfy

$$
\begin{array}{r}
\frac{1}{2}\left|\left\langle\sigma, \sigma_{d R T}\right\rangle\right|_{q}^{2} \leq \frac{1}{2^{1-q}}\left\|h_{\mathcal{T}} f\right\|_{q, \Omega}^{q}+\frac{c(p)}{2^{1-q}} \max \left\{\frac{\left\|u_{3}\right\|_{p, \Omega}}{j_{1,1}} \operatorname{osc}(f, \mathcal{T})\right. \\
\left.+\frac{c(p)}{2^{2-q}} M\left\|I_{N C} u_{3}-u_{3}\right\|_{N C, 2, \Omega}^{2} C_{2} \cdot \operatorname{Osc}(f, \mathcal{T})\right\} .
\end{array}
$$

Proof. The choice $\alpha:=\sigma, \beta:=\prod_{0} \sigma_{d R T}=\sigma_{C R}$, and $b:=\nabla_{N C} u_{C R}$ in Lemma 3.3 leads to

$$
\left|\left\langle\sigma, \Pi_{0} \sigma_{d R T}\right\rangle\right|_{q}^{2}+c(p)\left(E^{*}(\sigma)-E_{d}^{*}\left(\sigma_{d R T}\right)\right) \leq-c(p) \int_{\Omega} \nabla_{N C} u_{C R} \cdot\left(\sigma-\Pi_{0} \sigma_{d R T}\right) d x .
$$

The conforming $\mathcal{P}_{3}$ companion $u_{3} \in \mathcal{P}_{3}(\mathcal{T}) \cap V$ with $u_{C R}=I_{N C} u_{3}$ from Lemma 3.2 shows

$$
\begin{aligned}
& -\int_{\Omega}\left(\sigma-\Pi_{0} \sigma_{d R T}\right) \cdot \nabla_{N C} u_{C R} d x \\
= & -\int_{\Omega}\left(\sigma-\sigma_{d R T}\right) \cdot \nabla_{N C}\left(I_{N C} u_{3}-u_{3}\right) d x-\int_{\Omega}\left(\sigma-\sigma_{d R T}\right) \cdot \nabla u_{3} d x \\
= & \int_{\Omega}\left(\sigma-\sigma_{d R T}\right) \cdot\left(I-\Pi_{0}\right) \nabla u_{3} d x+\int_{\Omega} u_{3} \operatorname{div}\left(\sigma-\sigma_{d R T}\right) d x .
\end{aligned}
$$

The combination of the preceding results reads

$$
\begin{aligned}
& \left|\left\langle\sigma, \sigma_{C R}\right\rangle\right|_{q}^{2}+c(p)\left(E^{*}(\sigma)-E_{d}^{*}\left(\sigma_{d R T}\right)\right) \\
\leq & c(p)\left(\int_{\Omega}\left(\sigma-\sigma_{d R T}\right) \cdot\left(I-\Pi_{0}\right) \nabla u_{3} d x-\int_{\Omega}\left(u_{3}-\Pi_{0} u_{3}\right)\left(I-\Pi_{0}\right) f d x\right) .
\end{aligned}
$$


The sum of (5.3) and (4.1) plus Theorem 3.1 show that

$$
\begin{aligned}
& \left|\left\langle\sigma, \sigma_{C R}\right\rangle\right|_{q}^{2}+c(p)\left|E^{*}(\sigma)-E_{d}^{*}\left(\sigma_{d R T}\right)\right| \\
\leq & c(p) \max \left\{F(u)-F_{h}\left(I_{N C} u\right), \int_{\Omega}\left(\sigma-\sigma_{d R T}\right) \cdot\left(I-\Pi_{0}\right) \nabla u_{3} d x-\int_{\Omega}\left(u_{3}-\Pi_{0} u_{3}\right)\left(I-\Pi_{0}\right) f d x\right\} .
\end{aligned}
$$

The inequality (5.1) implies

$$
\begin{gathered}
\left|\left\langle\sigma, \sigma_{d R T}\right\rangle\right|_{q}^{2} \leq \frac{1}{2^{1-q}}\left\|h_{\mathcal{T}} f\right\|_{q, \Omega}^{q}+\frac{c(p)}{2^{1-q}} \max \left\{F(u)-F_{h}\left(I_{N C} u\right), \int_{\Omega}\left(\sigma-\sigma_{d R T}\right) \cdot\left(I-\Pi_{0}\right) \nabla u_{3} d x\right. \\
\left.-\int_{\Omega}\left(u_{3}-\Pi_{0} u_{3}\right)\left(I-\Pi_{0}\right) f d x\right\} .
\end{gathered}
$$

A piecewise Poincaré inequality applies in the last term with the constant $h_{T} / j_{1,1}$ from [23]. This shows that

$$
-\int_{\Omega}\left(u_{3}-\Pi_{0} u_{3}\right)\left(f-\Pi_{0} f\right) d x \leq \frac{\left\|u_{3}\right\|_{p, \Omega}}{j_{1,1}} \operatorname{osc}(f, \mathcal{T}) .
$$

The Young inequality and $\Pi_{0} \nabla u_{3}=\nabla_{N C} I_{N C} u_{3}$ show that

$$
\begin{aligned}
& \frac{c(p)}{2^{1-q}} \int_{\Omega}\left(\sigma-\sigma_{d R T}\right) \cdot\left(I-\Pi_{0}\right) \nabla u_{3} d x \\
\leq & \frac{1}{2}\left|\left\langle\sigma, \sigma_{d R T}\right\rangle\right|_{q}^{2}+\left.\frac{c^{2}(p)}{2^{3-2 q}} \sup _{x \in \Omega}\left(|\sigma|^{2-q}+\left|\sigma_{d R T}\right|^{2-q}\right)||\left|I_{N C} u_{3}-u_{3}\right|\right|_{N C, 2, \Omega} ^{2} .
\end{aligned}
$$

Recall that $\Pi_{0}\left(-\partial E_{d}^{*}\left(\sigma_{d R T}\right)\right)=\nabla_{N C} u_{C R}$. This and $|\nabla u|^{p-2}=|\sigma|^{2-q},\left|\nabla u_{C R}\right|^{p-2}=\left|\sigma_{C R}\right|^{2-q}$ conclude the proof.

\section{Numerical experiments}

This section is devoted to the numerical investigation of the lowest-order schemes of NCFEM and dRT-MFEM for the $p$-Laplace Problem on square domain and L-shaped domain.

\subsection{Numerical realization}

The edge-oriented basis functions $\psi_{E}$ for any interior edge $E \in \mathcal{E}(\Omega)$ in the triangulation $\mathcal{T}$ and their enumeration $\psi_{1}, \cdots, \psi_{m}$ at hand allows for the representation $u_{C R}=\sum_{j=1}^{m} x_{j} \psi_{j}$ with the unknown coefficient vector $x=\left(x_{1}, \cdots, x_{m}\right)$. The data structures and the discrete Euler-Lagrange equations are realized as in [7] and then minimized with the Matlab standard function fminunc and default parameters and the input of $E_{N C}, D E_{N C}$, and $D^{2} E_{N C}$ at $x$. 


\subsection{A posteriori error control}

The numerical experiments concern the practical application of the a posteriori error estimates (4.4) and (5.2) and their efficiency. Denote the left-hand side (LHS) of the two estimates by LHS (4.4) and LHS(5.2). The guaranteed upper bounds (GUB) read

$$
\begin{aligned}
\operatorname{GUB}(4.4)= & c(p) \max \left\{F\left(u_{C R}-u_{3}\right)+C_{1}\left\|u_{C R}-u_{3}\right\|_{N C, 2, \Omega}^{2}, C_{2} \cdot \operatorname{osc}(f, \mathcal{T})\right\} ; \\
\operatorname{GUB}(5.2)= & \frac{1}{2^{1-q}}\left\|h_{\mathcal{T}} f\right\|_{q, \Omega}^{q} \\
& +\frac{c(p)}{2^{1-q}} \max \left\{\frac{\left\|u_{3}\right\|_{p, \Omega}}{j_{1,1}} \operatorname{osc}(f, \mathcal{T})+\frac{c(p)}{2^{2-q}} M\left\|I_{N C} u_{3}-u_{3}\right\|_{N C, 2, \Omega}^{2}, C_{2} \cdot \operatorname{osc}(f, \mathcal{T})\right\} .
\end{aligned}
$$

The triangulations are either uniform with successive red-refinement or with an adaptive mesh-refinement algorithm with initial mesh $\mathcal{T}_{0}$ and then, for any triangle $T$ of a triangulation $\mathcal{T}_{\ell}$ at level $\ell=0,1,2,3, \cdots$, set

$$
\eta^{2}(T)=\left\|I_{\mathrm{NC}} u_{3}-u_{3}\right\|_{\mathrm{NC}, 2, T}^{2}+\left\|h_{\mathcal{T}} f\right\|_{q, T}^{q} .
$$

Given all those contributions, mark some set $\mathcal{M}_{\ell}$ of triangles in $\mathcal{T}_{\ell}$ of minimal cardinality with the bulk criterion

$$
1 / 2 \sum_{T \in \mathcal{T}_{\ell}} \eta_{\ell}^{2}(T) \leq \sum_{T \in \mathcal{M}_{\ell}} \eta_{\ell}^{2}(T)
$$

The refinement of all triangles in $\mathcal{M}_{\ell}$ plus minimal further refinements to avoid hanging nodes lead to the triangulation $\mathcal{T}_{\ell+1}$ within the newest-vertex bisection. The choice of the refinement-indicator $\eta(T)$ is motivated by the convergence theory of adaptive mesh-refining algorithms e.g., in the review article [8] with further details on the meshrefinement. The convergence history plots display the left-hand sides LHS(4.4), LHS(5.2) and the upper bounds $G U B(4.4), G U B(5.2)$ as function of the number of degrees of freedom (ndof) in a log-log scale.

\subsection{Example 1}

Consider the $p$-Laplace Problem on the square domain $\Omega:=(0,1)^{2}$ with the exact solution

$$
u(r)=(p-1)(1 /(\sigma+2))^{1 /(p-1)}\left(1-r^{(\sigma+p) /(p-1)}\right) /(\sigma+p) \text { for }|x|=r
$$

and right-hand side $f(r)=r^{\sigma}$ for $p=4, \sigma=7$. The reference value for the minimal energy $E=0.082674$ stems from Aitken extrapolation.

Fig. 1 and Fig. 2 display the global upper bounds (GUB) and the corresponding error terms (LHS) of the estimates from (4.4), (5.2) as explained in Subsection 6.2 for uniform and adaptive mesh-refinement. Fig. 3 displays the corresponding sequences of triangulations generated by adaptive FEM for (4.4). The exact solution is smooth and hence 


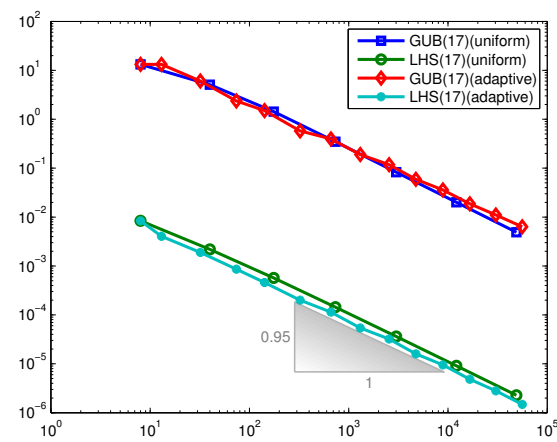

Figure 1: Convergence history of CR method on square domain.

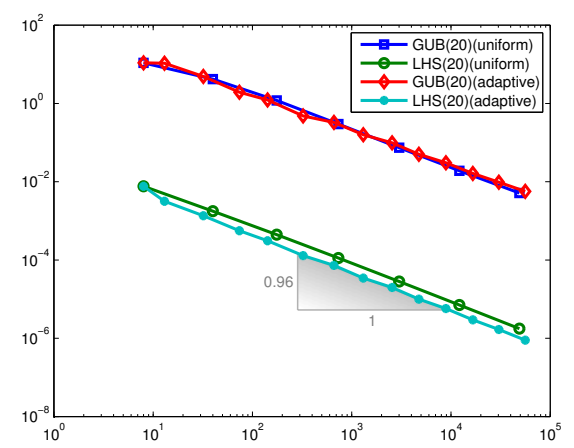

Figure 2: Convergence history of dRT method on square domain.
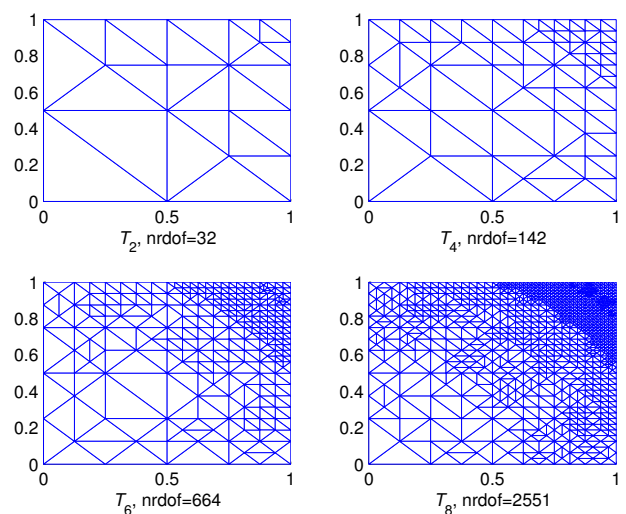

Figure 3: Adaptively generated triangulations $\mathcal{T}_{\ell}$ for $\ell=2,4,6,8$ on square domain.

uniform mesh-refining leads to optimal convergence rates (on structured grids with possible super convergence phenomena) and hence the adaptive mesh-refining is not necessarily better (on unstructured grids without higher symmetry). Lemma 3.2 implies that 
$\left\|u_{3}\right\|_{p, \Omega}$ is computable and is bounded by some generic constant.

\subsection{Example 2}

Consider the $p$-Laplace Problem on the $L$-shaped domain $\Omega:=[-1,1]^{2} \backslash(0,1] \times(0,-1]$ with $f \equiv 1$. The extrapolated energy reads $E=-0.34337$. Fig. 4 and Fig. 5 display the global upper bounds (GUB) and the corresponding error terms (LHS) of the estimates from (4.4), (5.2) for uniform and adaptive mesh-refinement. Fig. 6 displays the corresponding sequences of triangulations generated by adaptive FEM for (4.4). Since the constant righthand side $f \equiv 1$ leads to vanishing oscillations $\operatorname{osc}(f, \mathcal{T})=0$, the global upper bound in (4.4) and (5.2) is fully computable.

\subsection{Conclusions}

The proposed the dRT-MFEM of the $p$-Laplace problem is equivalent to CR-NCFEM. The numerical examples shows that the convergence results of CR-NCFEM and dRT-MFEM are consistent with the theoretical analysis.

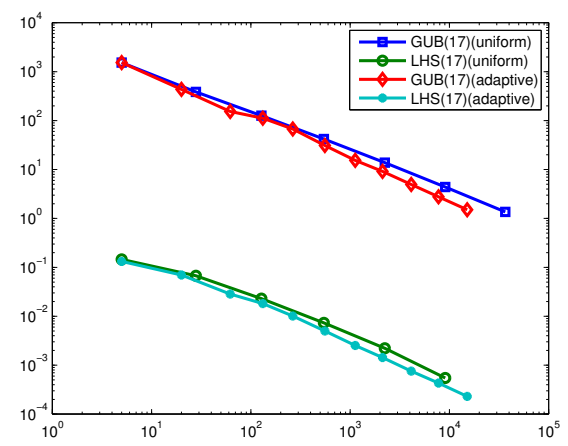

Figure 4: Convergence history of CR method on L-shaped domain.

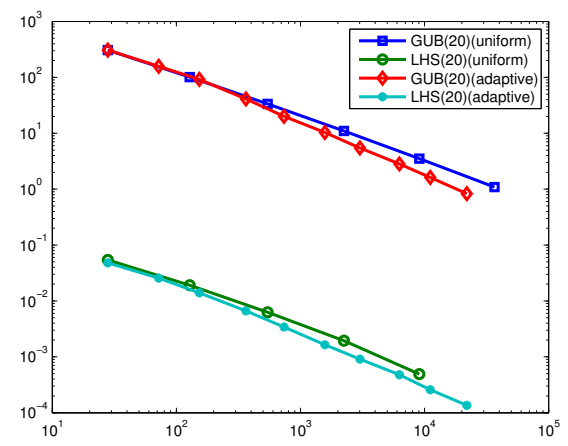

Figure 5: Convergence history of $\mathrm{dRT}$ method on $L$-shaped domain. 

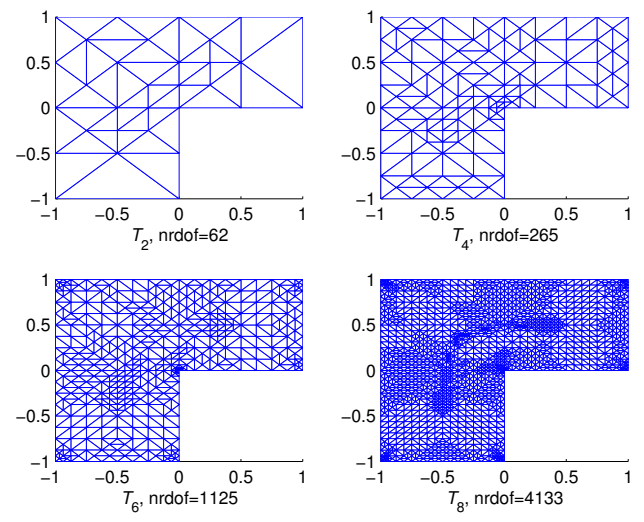

Figure 6: Adaptively generated triangulations $\mathcal{T}_{\ell}$ for $\ell=2,4,6,8$ on $L$-shaped domain.

\section{Acknowledgements}

This work was partly developed while the first author was visiting Hausdorff Research Institute of Mathematics in Bonn, Germany. We would like to thank professor C. Carstensen for his helpful suggestions. This author's research was supported by National Natural Science Foundation of China (Nos. 11571226, 11671313).

\section{References}

[1] R. A. Adams And J. F. Fournier, Sobolev Spaces, Second ed., Academic Press, 2003.

[2] T. ARBogast AND Z. X. CHEN, On the implementation of mixed methods as nonconforming methods for second-order elliptic problems, Math. Comput., 64 (1995), pp. 943-972.

[3] D. N. ARNOLD AND F. BREZZI, Mixed and nonconforming finite element methods: implementation, postprocessing and error estimates, RAIRO Modél. Math. Anal. Numér., 19 (1985), pp. 7-32 (1985).

[4] L. BeLENKI AND L. DienING, Optimality of an adaptive finite element method for the $p$-Laplacian equation, IMA. J. Numer. Anal., 32 (2012), pp. 484-510.

[5] S. C. BRENNER AND L. SCOTT, The Mathematical Theory of Finite Element Methods, Third ed., Texts in Applied Mathematics, 15, Springer, 2008.

[6] C. CARSTENSEN, Convergence of an adaptive FEM for a class of degenerate convex minimization problems, IMA. J. Numer. Anal., 28 (2008), pp. 423-439.

[7] C. CARSTENSEN AND C. BAhriaWATI, Three matlab implementations of the lowest-order Raviart-Thomas MFEM with a posteriori error control, CMAM., 5 (2005), pp. 333-361.

[8] C. Carstensen, M. Feischl, M. Page and D. Praetorius, Axioms of adaptivity, Comput. Methods Appl. Math., 67 (2014), pp. 1195-1253.

[9] C. CARSTENSEN AND D. GallistL, Guaranteed lower eigenvalue bounds for the biharmonic equation, Numer. Math., 126 (2014), pp. 33-51.

[10] C. CARSTEnSEn, J. Gedicke AND D. Rim, Explicit error estimates for Courant, CrouzeixRaviart and Raviart-Thomas finite element methods, J. Comput. Math., 30 (2012), pp. 337-353. 
[11] C. CARSTENSEN AND K. JOCHIMSEN, Adaptive finite element methods for microstructures? Numerical experiments for a 2-well benchmark, Computing, 71 (2003), pp. 175-204.

[12] C. CARSTENSEN AND R. KLOSE, A posteriori finite element error control for the $p$-Laplace problem, SIAM. J. Sci. Comput., 25 (2003), pp. 792-814.

[13] C. CARSTENSEN AND D. J. LIU, Nonconforming FEMs for an optimal design problem, SIAM. J. Numer. Anal., 53 (2015), pp. 874-894.

[14] C. CARSTENSEN, W. LIU AND N. N. YAN, A posteriori error control for $p$-Laplacian by gradient recovery in quasi-norm, Math. Comput., 75 (2006), pp. 1599-1616.

[15] P. G. CiARLET, The Finite Element Method for Elliptic Problems, North-Holland, 1978.

[16] E. CReuse, M. Farhloul AND L. Paquet, A posteriori error estimation for the dual mixed finite element method for the $p$-Laplacian in a polygonal domain, Comput. Methods Appl. Mech. Eng., 196 (2007), pp. 2570-2582.

[17] L. DienING AND F. ETTWEIN, Fractional estimates for non-differentiable elliptic systems with general growth, Forum Math., 3 (2008), pp. 523-556.

[18] M. FARHLOUL AND H. MANOUZI, On a mixed finite element method for the $p$-Laplacian, Canad. Appl. Math., 8 (2000), pp. 67-78.

[19] K. Y. KIM, Error estimates for a mixed finite volume method for the p-Laplacian problem, Numer. Math., 101 (2005), pp. 121-142.

[20] W. B. LIU AND J. W. BARRETT, Finite element approximaton of some degenerate monotone quasilinear elliptic system, SIAM J. Numer. Anal., 33 (1996), pp. 88-106.

[21] W. B. LIU AND N. N. YAN, Quasi-norm a priori and a posteriori error estimates for the nonconforming approximation of p-Laplacian, Numer. Math., 89 (2001), pp. 341-378.

[22] W. B. LIU AND N. N. YAN, On quasi-norm interpolation error estimation and a posteriori error estimates for p-Laplacian, SIAM J. Numer. Anal., 40 (2002), pp. 1870-1895.

[23] R. S. LAUgeSEN AND B. A. SiUdeJA, Minimizing neumann fundamental tones of triangles: an optimal Poincaré inequality, J. Differ. Equations, 249 (2010), pp. 118-135.

[24] L. D. MARINI, An inexpensive method for the evaluation of the solution of the lowest order RaviartThomas mixed method, SIAM J. Numer. Anal., 22 (1985), pp. 493-496.

[25] C. PADRA, A posteriori error estimators for nonconforming approximation of some quasiNewtonian flows, SIAM J. Numer. Anal., 34 (1997), pp. 1600-1615.

[26] R. T. Rockafellar, Convex Analysis, Princeton University Press, 1970.

[27] P. L. TAllec, Numerical Analysis of Viscoelastic Problems, Rech. Math. Appl., 15, Masson, Springer-Verlag, 1990.

[28] R. VERFÜRTH, A posteriori error estimates for nonlinear problems, Finite element discretizations of elliptic equations, Math. Comput., 62 (1994), pp. 445-475. 